\title{
NUTRITIONAL ASSESSMENT OF AN OBESE GROUP IN RELATION TO HEALTH IMPLICATION
}

\author{
By \\ Fatma El - Zahraa El - Sherif \\ Mohamed Samir El - Dashlouty \\ Nutrition \& Food Science Dept., \\ Faculty of Home Economics, \\ Minufiya, University \\ Nutrition \& Food Science Dept., \\ Faculty of Home Economics, \\ Minufiya, University \\ Amal Ibrahim Egela Abd El - Lateef \\ Nutrition \& Food Science Dept., \\ Faculty of Home Economics, Minufiya, University
}

Research Gournal Specific Fducation

Faculty of Specific Education

SMansoura University

ISSUE NO. 32, OCTOBER. 2013

مجلة بحوث التربية النوعية ـ جامعة المنصورة

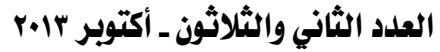




\title{
NUTRITIONAL ASSESSMENT OF AN OBESE GROUP IN RELATION TO HEALTH IMPLICATION
}

\author{
Fatma El-Zahraa El-Sherif" Mohamed Samir El-Dashlouty \\ Amal Ibrahim Egela Abd El - Lateef
}

\section{ABSTRACT}

Background: Underweight is a term describing a human whose A total number of 40 patients visiting Internist Department of Kasr El - Ainy Hospital (a governmental hospital) in Cairo were selected for present study provided that they wore overweight or obese based on BMI. Female and male patients were at the ages of $45-<55$ and $55-65$ years. The weight and length of each participant measured, and BMI calculated. Data of present work collected with interviews using questionnaire composed of several forms. The 24 hours recall method used to record food intake, which was analyzed to obtain levels of nutrients. Serum biochemical and haematological analyses were also carried out.

Prevalence of diabetes mellitus (DM) was much greater for females than males and raised with age. At $55-65$ years old $50-70 \%$ were inflicted with high blood pressure, at this age group $50 \%$ of males showed low efficiency of thyroid gland being possible a cause of obesity. This accompanied by low T.S.H. With increasing of age overweight, morbid and super obesity increased on account of class II obesity. TSF, AC and AMC increased also with the development of obesity. Total calories, protein, fat and carbohydrates intakes were higher than DRI. All studied biochemical analyses except uric acid and possibly HDL indicated health disorders. Haematological analyses confirmed the deteriorated health status of overweight and obese subjects of present work.

* Nutrition \& Food Science Dept., Faculty of Home Economics, Minufiya, University 


\section{INTRODUCTION}

Obesity increases the likelihood of various diseases, particularly heart disease, type 2 diabetes, obstructive sleep apnea, certain types of cancer disorders of liver and kidneys functions and osteoarthritis (Haslam and James, 2009 ). Obesity is most commonly caused by a combination of excessive food energy intake, lack of physical activity, and genetic susceptibility (Adams and Murphy, 2000 ).

Dieting and physical exercise are the mainstays of treatment for obesity. It is important to improve diet quality by reducing the consumption of energy - dense foods such as those high in fat and sugars, and by increasing the intake of dietary fiber. To supplement this, or in case of failure, anti - obesity drugs may be taken to reduce appetite or inhibit fat absorption. In severe cases, surgery is performed or an intragastric balloon is placed to reduce stomach volume and / or bowel length, leading to earlier satiation and reduced ability to absorb nutrients from food (Sweeting, 2007).

This work was carried out to assess the nutritional and health status of males and females, 45 - 65 years old, visiting the Internist Department, Kasr El - Ainy Hospital, Cairo.

\section{SUBJECTS AND METHODS}

\section{Sample:}

A total sample of 40 members enrolled in this study, being 10 females $\& 10$ males at the age of $45-<55$ years, and 10 females \& 10 males at the age of $55-65$ years. Study sample included only overweight or obese subjects based on body mass index (BMI ). Participants were the visitors of Internist Department, Kasr El - Ainy Hospital, Cairo.

\section{Instrumentation:}

The instrumentation of this study consisted of a structure interviewing questionnaire of different forms. Assessment of nutrient intake from food consumption data carried out at Faculty of Home Economics, Minufiya University (Shebin El - Kom) using the Counter Program for Nutrients of 
Ready to Eat Egyptian Food Version 1, in the Unit of Statistics and Food Analysis.

To evaluate results of total Kcal, DRI (2007). Equation for estimated energy requirements (EER) used. To evaluate results of other macronutrients, minerals and vitamins tables of Dietary Reference Intake (DRI, 2007) were used.

Anthropometric measurements were carried out as described by Jelliffee (1966), while biochemical analysis carried out using methods as follows: Serum glucose (Tietz, 1976), serum glutamate pyruvate transaminase (GPT, ALT) (Srivastava et al., 2002), creatinine (Chary and Sharma (2004), urea (Henery et al., 1974), uric acid (Fossati et al., 1980), TC (White et al., 1970 ), TG (Fossati and Pricipe (1982), VLDL " TG/5 " (Srivastava et al., 2002), LDL " TC - [TG + HDL] " was calculated according to (Castelli, 1977), HDL (Richmond, 1973), Hemoglubin (Jacobs et al., 2001 ), thyroid stimulating hormone (Waada et al., 1983), hemoglobin parameters " HCT, WBC, MCV, RBC, MCH, MCHC, RDW, PLT, PCT, MPV, PDW, Lym, Mon and GRA " respectively complete blood picture determined in the Laboratory of the Specific Misr \& El - Sudan Hospital, Ain Shams University, Cairo. Statistical analysis carried out using SPSS (1998), PC Statistical Software (Version 10, SPSS INC, Chicago, USA) in the Unit of Statistical and Food Analysis, Faculty of Home Economics, Minufiya University (Shebin El - Kom).

\section{RESULTS AND DISCUSSION}

\section{Percent distribution of study sample according to ailments:}

From results of tables $(1 \& 2)$ it is evident that prevalence of diabetes mellitus (DM) was much greater for females than males and raised with age; for the higher age nearly all participants $(90 \%)$ were diabetic. High blood pressure also increased with age; at $55-65$ years old $50-70 \%$ were affected by this disease. From deficiency anemia and kidney disorders were pronounced at the higher age $(20-30 \%)$ and $(30-60 \%)$ respectively. Low efficiency of thyroid gland was marked at the higher age, specially for males $(50 \%)$. It is known that low efficiency of thyroid gland (thyroidism) reduce 
the secretion of leptin; leptin is responsible for suppression of appetite and enhancement of energy expenditure (possibly reducing obesity) (Barness et al., 2007 ).

Table (1): Percent distribution of obese females \& males ( $45-<55$ years) according to morbidity

\begin{tabular}{|c|c|c|c|c|c|}
\hline \multirow[t]{3}{*}{ Variables } & \multicolumn{4}{|c|}{ Age $45-<55$ years } & \multirow[t]{3}{*}{$P$ value } \\
\hline & \multicolumn{2}{|c|}{$\begin{array}{l}\text { Females } \\
(\mathrm{N}=10)\end{array}$} & \multicolumn{2}{|c|}{$\begin{array}{l}\text { Males } \\
(\mathrm{N}=10)\end{array}$} & \\
\hline & No. & $\%$ & No. & $\%$ & \\
\hline \multicolumn{6}{|l|}{ Diabetes mellitus } \\
\hline Yes & 6 & 60 & 1 & 10 & $0.008 *$ \\
\hline No & 4 & 40 & 9 & 90 & \\
\hline \multicolumn{6}{|l|}{ Liver problems } \\
\hline Yes & 0 & 0 & 1 & 10 & $0.29 \mathrm{NS}$ \\
\hline No & 10 & 100 & 9 & 90 & \\
\hline \multicolumn{6}{|l|}{ High blood pressure } \\
\hline Yes & 2 & 20 & 2 & 20 & $1.00 \mathrm{NS}$ \\
\hline No & 8 & 80 & 8 & 80 & \\
\hline \multicolumn{6}{|c|}{ Iron deficiency anemia } \\
\hline Yes & 1 & 10 & 0 & 0 & $0.29 \mathrm{NS}$ \\
\hline No & 9 & 90 & 10 & 100 & \\
\hline \multicolumn{6}{|l|}{ Kidney disorders } \\
\hline Yes & 1 & 10 & 0 & 0 & $0.29 \mathrm{NS}$ \\
\hline No & 9 & 90 & 10 & 100 & \\
\hline \multicolumn{6}{|l|}{ High fat blood } \\
\hline Yes & 1 & 10 & 5 & 50 & $0.32 *$ \\
\hline No & 9 & 90 & 5 & 50 & \\
\hline
\end{tabular}

* Significant $\mathrm{P}<0.05$

** High significant $\mathrm{P}<0.01$

$* * *$ Very high significant $\mathrm{P}<0.001$

NS: Nonsignificant 


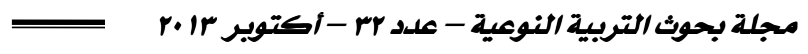

Table (2): Percent distribution of obese females \& males ( 55 - 65 years) according to morbidity

\begin{tabular}{|c|c|c|c|c|c|}
\hline \multirow[t]{3}{*}{ Variables } & \multicolumn{4}{|c|}{ Age $55-65$ years } & \multirow[t]{3}{*}{$\mathrm{P}$ value } \\
\hline & \multicolumn{2}{|c|}{$\begin{array}{l}\text { Females } \\
(\mathrm{N}=10)\end{array}$} & \multicolumn{2}{|c|}{$\begin{array}{c}\text { Males } \\
(\mathrm{N}=10)\end{array}$} & \\
\hline & No. & $\%$ & No. & $\%$ & \\
\hline \multicolumn{6}{|l|}{ Diabetes mellitus } \\
\hline Yes & 9 & 90 & 9 & 90 & $1.00 \mathrm{NS}$ \\
\hline No & 1 & 10 & 1 & 10 & \\
\hline \multicolumn{6}{|l|}{ Liver problems } \\
\hline Yes & 2 & 20 & 0 & 0 & $0.29 \mathrm{NS}$ \\
\hline No & 8 & 80 & 10 & 100 & \\
\hline \multicolumn{6}{|l|}{ High blood pressure } \\
\hline Yes & 5 & 50 & 7 & 70 & $1.00 \mathrm{NS}$ \\
\hline No & 5 & 50 & 3 & 30 & \\
\hline \multicolumn{6}{|l|}{ Iron deficiency anemia } \\
\hline Yes & 2 & 20 & 3 & 30 & $0.62 \mathrm{NS}$ \\
\hline & 8 & 80 & 7 & 70 & \\
\hline \multicolumn{6}{|l|}{ Kidney disorders } \\
\hline Yes & 3 & 30 & 6 & 60 & $0.69 \mathrm{NS}$ \\
\hline No & 7 & 70 & 4 & 40 & \\
\hline \multicolumn{6}{|l|}{ High fat blood } \\
\hline Yes & 6 & 60 & 5 & 50 & $0.035^{*}$ \\
\hline & 4 & 40 & 5 & 50 & \\
\hline \multicolumn{6}{|l|}{ Low efficiency of thyroid gland } \\
\hline Yes & 1 & 10 & 5 & 50 & $0.31 \mathrm{NS}$ \\
\hline No & 9 & 90 & 5 & 50 & \\
\hline \multicolumn{6}{|l|}{ Ulcer } \\
\hline Yes & 1 & 10 & 0 & 0 & $1.00 \mathrm{NS}$ \\
\hline No & 9 & 90 & 10 & 100 & \\
\hline \multicolumn{6}{|l|}{ Rheumatism } \\
\hline Yes & 1 & 10 & 1 & 10 & $1.00 \mathrm{NS}$ \\
\hline & 9 & 90 & 9 & 90 & \\
\hline \multicolumn{6}{|l|}{ Roughness of the joints } \\
\hline Yes & 1 & 10 & 1 & 10 & $1.00 \mathrm{NS}$ \\
\hline No & 9 & 90 & 9 & 90 & \\
\hline \multicolumn{6}{|l|}{$*$ Significant $\mathrm{P}<0.05$} \\
\hline$* * *$ Very high significant $\mathrm{P}<0$ & & NS: & Nonsi & gnific: & \\
\hline
\end{tabular}




\section{Frequency distribution of sample among weight classification groups :}

Results of tables ( $3 \& 4$ ) revealed that with increasing of age overweight, morbid obesity and super obesity increased on account of class II obesity. The total sample showed that overweight was at $40 \%$ level while obesity $60 \%$ being class I obesity $35 \%$, class II obesity $35 \%$ \& morbid obesity $15 \%$.

Table (3) : Frequency distribution of females among weight classification groups*

\begin{tabular}{||c|c|c|c|c|c|c||}
\hline \multirow{2}{*}{ Parameters } & \multicolumn{2}{|c|}{$\begin{array}{c}45-<5 \text { years } \\
(\text { No. 10 ) }\end{array}$} & \multicolumn{2}{c|}{$\begin{array}{c}55-65 \text { years } \\
(\text { No. 10 ) }\end{array}$} & \multicolumn{2}{c||}{$\begin{array}{c}45 \text {-65 years } \\
(\text { No. 20 ) }\end{array}$} \\
\cline { 2 - 7 } & No. & $\%$ of group & No. & $\%$ of group & No. & $\%$ of group \\
\hline \hline Overweight & 2 & 20 & 3 & 30 & 5 & 25 \\
Class I obesity & ----- & ---- & ---- & ---- & ---- & ---- \\
Class II obesity & 4 & 40 & ---- & --- & 4 & 20 \\
Morbid obesity & 2 & 20 & 3 & 30 & 5 & 25 \\
Supper obesity & 2 & 20 & 4 & 40 & 6 & 30 \\
\hline
\end{tabular}

*Classification based on BMI (kg/m2) level: < 18.5

Underweight; $12.5-24 \mathrm{~g}$

Normal; $25-29 \mathrm{~g}$

Overweight; $30-34 \mathrm{~g}$

Class I obesity; 35 - $39 \mathrm{~g}$

Class II obesity; 40 - $44 \mathrm{~g}$

Morbid obesity; 45 - 50 g Supper obesity (Mokdad et al., 2004 and Imaz et al., 2008 ).

Table (4): Frequency distribution of males among weight classification groups*

\begin{tabular}{|c|c|c|c|c|c|c||}
\hline \multirow{2}{*}{ Parameters } & \multicolumn{2}{|c|}{$\begin{array}{c}45-<55 \text { years } \\
(\text { No. 10 ) }\end{array}$} & \multicolumn{2}{c|}{$\begin{array}{c}55-65 \text { years } \\
(\text { No. } 10)\end{array}$} & \multicolumn{2}{c|}{$\begin{array}{c}45-65 \text { years } \\
\text { No. 20 ) }\end{array}$} \\
\cline { 2 - 7 } & No. & $\%$ of group & No. & $\%$ of group & No. & $\%$ of group \\
\hline \hline Overweight & 4 & 40 & 4 & 40 & 8 & 40 \\
Class I obesity & 4 & 40 & 3 & 30 & 7 & 35 \\
Class II obesity & 2 & 20 & ---- & ---- & 2 & 10 \\
Morbid obesity & ---- & ---- & 3 & 30 & 3 & 15 \\
Supper obesity & ---- & ---- & ---- & ---- & ---- & ---- \\
\hline
\end{tabular}

*Classification based on BMI $(\mathrm{kg} / \mathrm{m} 2)$ level: $<18.5$ 


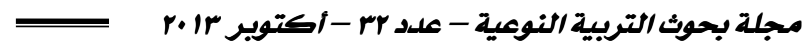

Underweight; $12.5-24 \mathrm{~g}$

Normal; $25-29 \mathrm{~g}$

Overweight; 30 - $34 \mathrm{~g}$

Class I obesity; 35 - $39 \mathrm{~g}$

Class II obesity; $40-44 \mathrm{~g}$

Morbid obesity; 45 - 50 g Supper obesity (Mokdad et al., 2004 and Imaz et al., 2008 ).

Data of table (5) revealed with increasing of age TSF increased nonsignificantly for females and males. AC and AMC also increased which was significantly for females and nonsignificantly for males. This also confirmed the more development of overweight and obesity with age.

Table (5) : Some anthropometric measurement of females and male $45-<55 \& 55$

-65 years of age

\begin{tabular}{||c|c|c|c|c||}
\hline \multirow{2}{*}{ Parameters } & \multicolumn{2}{|c|}{ Females } & \multicolumn{2}{c||}{ Males } \\
\cline { 2 - 5 } & $45-<55$ years & $55-65$ years & $45-<55$ years & $55-65$ years \\
\hline \hline TSF mm & 3.42 & $3.50 \mathrm{NS}$ & 3.28 & $3.43 \mathrm{NS}$ \\
AC cm & 39.8 & $43.67^{*}$ & 39.6 & $40.33 \mathrm{NS}$ \\
AMC cm & 38.73 & $42.57^{*}$ & 38.57 & $39.25 \mathrm{NS}$ \\
\hline
\end{tabular}

TSF

$\mathrm{AMC}=\mathrm{AC}-\left(3.14 \frac{\boldsymbol{X}}{\mathbf{1 0}}\right)$.

* Significant at $\mathrm{P}<0.05$ NS: Nonsignificant at $\mathrm{P}<0.05$.

\section{Macronutrients of food as estimated for participants:}

Data of tables $(6 \& 7)$ revealed that intakes calories, protein, fat and carbohydrates by $45-<55$ and $55-65$ years were higher than DRI recommendation, except fat of younger males (98.67 \% of DRI ). For both age groups nonsignificant difference found between intakes of females and males. Meanwhile numerically total calories and carbohydrates were higher for females than males at both age groups. The higher total calories, protein, fat and carbohydrates (energy sources) that DRI may be the reason for overweight and obesity of study sample. 
Table (6) : Macronutrients of food as estimated for $45-<55$ years old participants

\begin{tabular}{|c|c|c|c|c|c|c|c|c|c|c|c|}
\hline \multirow[b]{2}{*}{ Variables } & \multicolumn{5}{|c|}{ Females } & \multicolumn{5}{|c|}{ Males } & \multirow{2}{*}{$\begin{array}{c}P \\
\text { value }\end{array}$} \\
\hline & $\begin{array}{c}\text { Mean }+ \\
\text { SD }\end{array}$ & Min & $\operatorname{Max}$ & $D R I$ & $\begin{array}{l}\% \text { of } \\
\text { DRI }\end{array}$ & $\begin{array}{c}\text { Mean + } \\
\text { SD }\end{array}$ & Min & $\operatorname{Max}$ & DRI & $\begin{array}{l}\% \text { of } \\
\text { DRI }\end{array}$ & \\
\hline Kcal & $\begin{array}{c}4065+ \\
874\end{array}$ & 3274 & 5664 & 2444.95 & 167.64 & $\begin{array}{r}3723.90 \\
+984 \\
\end{array}$ & 2512 & 4728 & 2767.87 & 134.55 & $\begin{array}{c}0.58 \\
N S\end{array}$ \\
\hline $\begin{array}{c}\text { Protein (g / } \\
\text { day) }\end{array}$ & $\begin{array}{c}151.80+ \\
49.6\end{array}$ & 104.60 & 220 & 46 & 414.57 & $\begin{array}{c}144.60+ \\
36.45 \\
\end{array}$ & 104.80 & 190.70 & 56 & 258.21 & $\begin{array}{l}0.82 \\
N S\end{array}$ \\
\hline Fat (g/day) & $\begin{array}{c}106.6+ \\
59.3\end{array}$ & 42.40 & $\begin{array}{c}173.7 \\
0 \\
\end{array}$ & 81.50 & 130.80 & $\begin{array}{c}93.50+ \\
61.9\end{array}$ & 41.5 & 199.9 & 92.26 & 98.67 & $\begin{array}{c}0.79 \\
N S \\
\end{array}$ \\
\hline $\begin{array}{c}\text { Carbohy - } \\
\operatorname{drates}(\mathrm{g} / \text { day })\end{array}$ & $\begin{array}{c}633.00+ \\
126.2 \\
\end{array}$ & 502 & 804 & 381.86 & 165.77 & $\begin{array}{c}576.00+ \\
152\end{array}$ & 362 & 712 & 428.36 & 34.47 & $\begin{array}{c}0.59 \\
N S\end{array}$ \\
\hline
\end{tabular}

Table (7) : Macronutrients of food as estimated for $55-65$ years old participants

\begin{tabular}{|c|c|c|c|c|c|c|c|c|c|c|c|}
\hline \multirow[b]{2}{*}{ Variables } & \multicolumn{5}{|c|}{ Females } & \multicolumn{5}{|c|}{ Males } & \multirow[t]{2}{*}{$P$ value } \\
\hline & $\begin{array}{c}\text { Mean + } \\
\text { SD }\end{array}$ & Min & $\operatorname{Max}$ & $D R I$ & $\begin{array}{l}\% \text { of } \\
D R I\end{array}$ & $\begin{array}{c}\text { Mean }+ \\
S D\end{array}$ & Min & $\operatorname{Max}$ & $D R I$ & $\begin{array}{l}\% \text { of } \\
D R I\end{array}$ & \\
\hline Kcal & $\begin{array}{c}3720.50 \\
+599 \\
\end{array}$ & 2747 & 43.04 & 2454.59 & 151.58 & $\begin{array}{c}3597.00 \\
+807 \\
\end{array}$ & 2386 & 4530 & 2682.07 & 134.11 & $0.63 \mathrm{NS}$ \\
\hline $\begin{array}{l}\text { Protein } \\
\text { (g/day) }\end{array}$ & $\begin{array}{r}156.30 \\
+21.8\end{array}$ & 127.90 & 189.60 & 46 & 339.78 & $\begin{array}{r}162.50 \\
+48.3\end{array}$ & 105.50 & 248.30 & 56 & 290.18 & $0.78 \mathrm{NS}$ \\
\hline Fat (g/day) & $\begin{array}{c}81.30+ \\
12.6 \\
\end{array}$ & 67.50 & 101.50 & 81.82 & 99.37 & $\begin{array}{c}95.50+ \\
26.4 \\
\end{array}$ & 47.90 & 127.30 & 89.40 & 106.82 & $0.25 \mathrm{NS}$ \\
\hline $\begin{array}{c}\text { Carbohy - } \\
\text { drates (g / day) }\end{array}$ & $\begin{array}{r}590.90 \\
+130.2 \\
\end{array}$ & 393 & 733 & 383.54 & 154.07 & $\begin{array}{r}576.00 \\
+147\end{array}$ & 297.80 & 690.70 & 413.37 & 126.26 & $0.38 \mathrm{NS}$ \\
\hline
\end{tabular}

\section{Minerals of food as estimated for participants:}

The results of table (8) showed that intakes of Ca was lower while Fe and $\mathrm{Na}$ higher by both age groups compared to DRI. The low $\mathrm{Ca}$ intake is no proper for bones health, pronounced Fe increase may affect the function of liver, while high $\mathrm{Na}$ intake cause high blood pressure and other diseases (Whitney, Eleanor et al., 1991 ). 


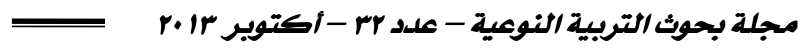

Table (8) : Minerals of food as estimated for $45-<55$ and 55 - 65 years old participants

\begin{tabular}{|c|c|c|c|c|c|c|c|c|c|c|c|}
\hline \multirow[b]{2}{*}{ Variables } & \multicolumn{5}{|c|}{ Females $(45-<55)$} & \multicolumn{5}{|c|}{ Males $(45-<55)$} & \multirow[t]{2}{*}{$P$ value } \\
\hline & $M e a n+S D$ & Min & $\operatorname{Max}$ & $D R I$ & $\begin{array}{l}\% \text { of } \\
\text { DRI }\end{array}$ & $M e a n+S D$ & Min & $\operatorname{Max}$ & $D R I$ & $\begin{array}{l}\text { \% of } \\
\text { DRI }\end{array}$ & \\
\hline $\mathrm{Ca}(\mathrm{mg} / \mathrm{day})$ & $1125+203$ & 907 & 1364 & 1200 & 93.75 & $800.7+310$ & 396.9 & 1151 & 1200 & 66.73 & $0.13 \mathrm{NS}$ \\
\hline$F e(m g / d a y)$ & $14.3+2.7$ & 11.6 & 17.2 & 8 & 178.75 & $12.9+4.0$ & 10.1 & 18.9 & 8 & 161.25 & $0.60 \mathrm{NS}$ \\
\hline \multirow[t]{2}{*}{$\mathrm{Na}(\mathrm{mg} / \mathrm{day})$} & $2381+1239$ & 743 & 3339 & 500 & 476.2 & $1830+1458$ & 594.3 & 3935 & 500 & 118.86 & $0.57 \mathrm{NS}$ \\
\hline & \multicolumn{5}{|c|}{ Females $(55-65)$} & \multicolumn{5}{|c|}{ Males $(55-65)$} & \\
\hline Ca (mg / day ) & $1021+34$ & 562.2 & 1564 & 1200 & 85.08 & $1148+297$ & 545 & 1561 & 1200 & 95.67 & $0.47 \mathrm{NS}$ \\
\hline$F e(m g / d a y)$ & $13.9+3.7$ & 10.1 & 19.3 & 8 & 173.75 & $13.5+3.8$ & 10.9 & 21.9 & 8 & 168.75 & $0.85 \mathrm{NS}$ \\
\hline $\mathrm{Na}$ (mg / day & $1419.9+30$ & 992 & 1775 & 500 & 283.98 & $2248+1119$ & 997 & 384 & 500 & 449.6 & $0.11 \mathrm{NS}$ \\
\hline
\end{tabular}

\section{Vitamins of food as estimated for participants:}

Data of table (9) revealed that no deficiency was found for vitamins intakes by study sample. This was in line with the results of Abo - Tabl (2005 ). Nevertheless as reported by Whitney, Eleanor and Rolfes, Sharon (1993) except of B vitamins, C and other vitamins mat be undesirable for health. 
Table (9) : Vitamins of food as estimated for $45-<55$ and $55-65$ years old participants

\begin{tabular}{|c|c|c|c|c|c|c|c|c|c|c|c|}
\hline \multirow[b]{2}{*}{ Variables } & \multicolumn{5}{|c|}{ Females $(45-<55)$} & \multicolumn{5}{|c|}{ Males $(45-<55)$} & \multirow[t]{2}{*}{ P value } \\
\hline & $M e a n+S D$ & Min & $\operatorname{Max}$ & $D R I$ & $\begin{array}{l}\% \text { of } \\
\text { DRI }\end{array}$ & $M e a n+S D$ & Min & $\operatorname{Max}$ & $D R I$ & $\begin{array}{l}\% \text { of } \\
\text { DRI }\end{array}$ & \\
\hline B1 (mg / day & $2.5+1.2$ & 1.4 & 3.7 & 1.1 & 227.27 & $1.9+0.5$ & 1.5 & 2.7 & 1.2 & 158.33 & $0.47 \mathrm{NS}$ \\
\hline$B 2(m g / d a y)$ & $2.9+1.4$ & 1.3 & 4.6 & 1.1 & 263.64 & $2.7+1.2$ & 1.02 & 5.5 & 1.3 & 207.69 & $0.79 \mathrm{NS}$ \\
\hline Niacin (mg / day) & $42.7+23.1$ & 16 & 71.9 & 14 & 305 & $32.8+15.2$ & 17.3 & 51.1 & 16 & 205 & $0.50 \mathrm{NS}$ \\
\hline$A(\mu R E)$ & $1661+294$ & 491 & 2191 & 700 & 237.29 & $2014+850$ & 1317 & 3247 & 900 & 223.78 & $0.57 \mathrm{NS}$ \\
\hline \multirow[t]{2}{*}{$C(m g / d a y)$} & $138.7+24.1$ & 116 & 166 & 75 & 184.93 & $98.3+48.4$ & 33.8 & 150.6 & 90 & 109.22 & $0.19 \mathrm{NS}$ \\
\hline & \multicolumn{5}{|c|}{ Females $(55-65)$} & \multicolumn{5}{|c|}{ Males $(55-65)$} & \\
\hline B1 (mg / day & $2.0+0.53$ & 1.5 & 2.7 & 1.1 & 181.82 & $2.2+0.65$ & 1.2 & 3.2 & 1.2 & 183.33 & $0.69 \mathrm{NS}$ \\
\hline$B 2(m g / d a y)$ & $2.8+0.86$ & 2.1 & 4.3 & 1.1 & 254.55 & $5.0+3.6$ & 2.1 & 11.0 & 1.3 & 384.61 & $0.17 \mathrm{NS}$ \\
\hline Niacin (mg / day) & $33.5+7.3$ & 20.9 & 42.9 & 14 & 239.29 & $33.7+11.1$ & 17.3 & 54.2 & 16 & 210.63 & $0.96 N S$ \\
\hline$A(\mu R E)$ & $1882+632$ & 1088 & 2770 & 700 & 268.86 & $2028+429.3$ & 1450 & 2624 & 900 & 225.33 & $0.61 \mathrm{NS}$ \\
\hline$C(m g / d a y)$ & $129.1+49.5$ & 44.9 & 191.5 & 75 & 172.1 & $104.9+46.5$ & 43.8 & 193.2 & 90 & 116.56 & $0.37 \mathrm{NS}$ \\
\hline
\end{tabular}

\section{Serum analysis:}

Results of table (10) indicated that except for uric acid and possibly HDL all other values for serum parameters showed health disorders which revealed by deviation from the range for normal, affected mean or Max and even sometimes the Min, confirming the deteriorated health of study sample which were overweight to obese including morbid and sample obesity. T.S.H. $(1.9+6.11)$ which was described by medical report for study as " Low " (giving no range for normal ). According to Barness et al., (2007) hypothyrodism (low T.S.H.) reduces the leptin hormone secretion (from that tissues ), which results in increase of food intake and decreased of energy expenditure leading accordingly to overweight and obesity. This was also reported by Rosen et al., (1993) and Flier (2004). 


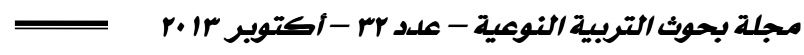

Table (10) : Biochemical analyses data of study sample $(\mathrm{N}=40)$

\begin{tabular}{|c|c|c|c|c||}
\hline Variable & Mean $+S D$ & Min & Max & Range \\
\hline \hline Fasting serum glucose $(\mathrm{FBS})(\mathrm{mg} / \mathrm{dl})$ & $182.89+69.50$ & 93 & 313 & $70-110$ \\
Serum glucose after 2 hours of $\mathrm{meal}(\mathrm{PPBS})(\mathrm{mg} / \mathrm{dl})$ & $255.28+105.52$ & 101 & 441 & $140-180$ \\
Serum $\mathrm{GPT}(\mathrm{U} / \mathrm{L})$ & $29.33+12.98$ & 12 & 55 & $19-52$ \\
Serum creatinine $(\mathrm{mg} / \mathrm{dl})$ & $0.87+0.20$ & 0.5 & 1.5 & $0.7-1.2$ \\
Urea $(\mathrm{mg} / \mathrm{dl})$ & $54.00+3.5$ & 22 & 70 & $15-35$ \\
Uric acid $(\mathrm{mg} / \mathrm{dl})$ & $4.6+0.9$ & 35 & 7.6 & $3.5-8.5$ \\
Total cholesterol $(\mathrm{TC})(\mathrm{mg} / \mathrm{dl})$ & $196.56+41.20$ & 125 & 269 & $\mathrm{Up}$ to 200 \\
Triglycerides $(\mathrm{TG})(\mathrm{mg} / \mathrm{dl})$ & $144.44+62.65$ & 57 & 308 & Up to 150 \\
High density lipoprotein cholesterol $(\mathrm{HDL})(\mathrm{mg} / \mathrm{dl})$ & $45.44+32.0$ & 32 & 57 & $39-96$ \\
Low density lipoprotein cholesterol $(\mathrm{LDL})(\mathrm{mg} / \mathrm{dl})$ & $121.62+59.52$ & 69 & 184 & $0-160$ \\
Thyroid stimulating hormone $(\mathrm{T} . \mathrm{S} . \mathrm{H}).(\mathrm{U} / \mathrm{U} / \mathrm{ml})$ & $1.9+6.11$ & 0.5 & 3.69 & $-\cdots--$ \\
\hline \hline
\end{tabular}

\section{Haematological analysis:}

Data of table (11) revealed the possible deteriorated of overweight and obese subjects of present study. This was indicated by low Min hemoglobin and hematocrit leading to possible risk of anemia which inflicted $20 \%$ of females and $30 \%$ of males at 55 - 65 age years old (Table 2 ). Disorders were found also for Min, Mix values, or sometime for both (as for MCV, MPV and Mon ). Moreover immunity cells a LYMM (low than Min) and monocytes (low Min and higher Max) were not indicative for good health. 
Table (20) : Haematological analyses data of study sample $(\mathrm{N}=40)$

\begin{tabular}{|c|c|c|c|c|}
\hline Variable & $M e a n+S D$ & Min & $\operatorname{Max}$ & Range \\
\hline Hemoglobin $(H b)(g / d l)$ & $12.84+2.4$ & 8.9 & 17.0 & $11-17$ \\
\hline Hematocrit (HCT) (\%) & $36.1+4.2$ & 28.1 & 40.7 & $35-51$ \\
\hline White blood cells $(\mathrm{WBC})(103 / \mathrm{mm} 3,103 \mathrm{ml})$ & $5.2+1.3$ & 3.1 & 6.8 & $4.5-11$ \\
\hline Mean corpuscular volume $(M C V)(f L)$ & $79.0+2.0$ & 38 & 122 & $80-100$ \\
\hline Red blood cells $(\mathrm{RBC})(106 / \mathrm{mm} 3,106 \mathrm{ml})$ & $4.79+0.9$ & 4.2 & 5.5 & $4.3-5.7$ \\
\hline Mean corpuscular hemoglobin $(\mathrm{MCH})$ & $27.5+1.1$ & 24.3 & 33.1 & $26-34$ \\
\hline Mean corpuscular hemoglobin concentration $(\mathrm{MCHC})(\mathrm{g} / \mathrm{dl})$ & $34.8+2.3$ & 25.1 & 45 & $31-37$ \\
\hline Red blood cell distribution width (RDW) (\%) & $13.4+1.8$ & 8.5 & 20.3 & $11.8-15$ \\
\hline Platelets $(106 / \mathrm{mm3}, 106 \mathrm{ml})$ & $234+5.6$ & 110 & 376 & $150-450$ \\
\hline Platelet crit volume $(P C T)(\%)$ & $0.222+0.04$ & 0.15 & 0.31 & $0.19-0.36$ \\
\hline Mean platelet volume $(M P V)(f L)$ & $9.1+0.8$ & 4.2 & 15.1 & $7.4-10.9$ \\
\hline Platelet diminution width (PDW) (\%) & $16.3+2.0$ & 11.5 & 22.2 & $15.5-17.1$ \\
\hline Lymphocytes (Lym) (\%) & $1.4+0.09$ & 1.0 & 2.0 & $1.3-2.9$ \\
\hline Monocytes (Mon) (\%) & $5.7+0.3$ & 1.4 & 12 & $1.7-9.3$ \\
\hline Granulocytes (GRA) (\%) & $67.7+0.5$ & 68 & 68.2 & $42.2-75.2$ \\
\hline
\end{tabular}

\section{REFERENCES}

- Abo - Tabl, A.M.R.G. (2005) : The Relationship Between Obesity, Physical Health and Some Psychological Variable for Secondary School Female Students In Menoufiya Governorate. Ph.D. Thesis, Menoufiya University.

- Adams, J.P. and Murphy, P.G. (2000) : "Obesity in anaesthesia and intensive care". Br. J. Anaesth., 85 (1) : 91 - 108.

- Barness, L.A.; Ooitz, J.M. and Gilbert - Barness, E. (2007) : "Obesity: Genetic, molecular and environmental aspects". Am. J. Med. Genet. A, 143A (24) : 3016 -34 .

- Castelli, W. (1977) : Determination of LDL. Slavo Diagnostics - Made in Italy. Circulation, $55: 767-769$.

- Chary, T.M. and Sharma, H. (2004) : Practical Biochemistry for Medical and Dental Students. Jaypce Brothers. Medical Published (p) LTD, New Delhi. 
- DRI (2007) : Dietary Reference Intakes for Energy, Carbohydrate, Fiber, Fat, Fatty Acids, Cholesterol, Protein and Amino Acids. This Repot may be accessed Via www.nap.edu.

- Flier, J.S. (2004) : "Obesity wars: Molecular progress confronts an expanding epidemic". Cell, $116(2): 337$ - 50.

- Fossati, P.; Principe, L. and Berti, G. (1980) : Enzymatic colorimetric method of determination of uric acid in serum. Clin. Chem., 26 (2) : 227 - 237.

- Fossati, P. and Principe, L. (1982) : Enzymatic colorimetric method of determination of triglycerides. Clin. Chem., $28: 2077$.

- Haslam, D.W. and James, W.P. (2009) : "Obesity: Lancet, 366 (9492 ): 1197 209.

- Henery, J.B.; Sanford, F.; Todd, G.F. and Davidson, B.W. (1974) : Clinical Diagnosis and Management by Laboratory Methods. 16 th Ed., W.S. Saunders Company, Philadelphia.

- Imaz, I.; Martinez - Cervell, C.; Garcia - Alvarez, E.E.; Sendra - Gutierrez, J.M. and Gonzalez - Enriquez, J. (2008) : "Safety and effectiveness of the intragastric balloon for obesity : A meta - analysis". Obes. Surg., 18 (7) : 841 - 846.

- Jacobs, B.S.; Oxley, D.K. and Demott, W.R. (2001) : Laboratory Text Handbook. Lexi. Comp., INC.

- Jelliffee, D.B. (1966) : The Assessment of the Nutritional of the Community. World Health Organization, Geneva.

- Mokdad, A.H.; Marks, J.S.; Stroup, D.F. and Gerberding, J.L. (2004) : "Actual causes of death in the United States, 2000" (PDF ). JAMA, 291 (10) : $1238-45$.

- Richmond, N. (1973) : Colorimetric method of determination of total cholesterol and high density lipoprotein cholesterol (HDL). Clin. Chem., 19 : 1350 - 1356.

- Rosen, T.; Bosaeus, I.; Tolli, J.; Lindstedt, G. and Bengtsson, B.A. (1993) : "Increased body fat mass and decreased extracellular fluid volume in adults with growth hormone deficiency". Clin. Endocrinol. (Oxf ), 38 (1) : 63 - 71.

- SPSS (1998) : Statistical Package For Social Science, Computer Software, Ver. 10, SPSS Company. London, UK.

- Srivastava, L.M., Das, N. and Sinha, S. (2002) : Essential of Practical Biochemistry. CBC Publishers and Distributors. USA. 
- Tietz, N.W. (1976) : Fundamentals of Clinical Chemistry. W.B. Saunders, Philadelphia. 243.

- Waada, H.G.; Danisch, R.J.; Baxter, S.R.; Federici, M.M.; Fraser, R.C.; Brownmiller, L.J. and Lankford, J.C. (1983) : Enzyme immunoassay of glycoprotein topic hormones - choriogon - adotropin, leutropin, thyrotropin with solid phase monoclonal antibody for the alpha - subunit and enzyme - coupled monoclonal antibody specific for the beta - subunit. Clin. Chem., 28 : 1862 1866.

- White, B.; Erickson, M. and Stevens, S. (1970) : Determination of Total Cholesterol. Bicon Diagnostics - Made in Germany. Chemistry For Medical Technologists, Saint Louis.

- Whitney, Eleanor N.; Cataldo, Corinne, B. and Rolfes, Sharon R. (1991) : Understanding Normal and Clinical Nutrition. West Publishing Company, New York, Los Angeles, San Francisco.

- Whitney, Eleanor N. and Rolfes, Sharon R. (1993): Understanding Nutrition. West Publishing Company, New York, Los Angeles, San Francisco.

- Sweeting, H.N. (2007) : "Measurement and definitions of obesity in childhood and adolescent: A field guide for the uninitiated". Nutr. J., 6 (1) : 32. 


\section{تقييم الحالة الغذائية لمبموعة هن الصصابين بالسمنة}

\section{فى ارتباط هـ التأثيرات الصهية}

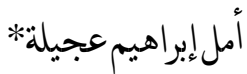

أ .د ـ محمد سمير الدشلوطر*

أ .د ـ فاطمة الزهراء الشريف

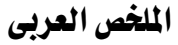

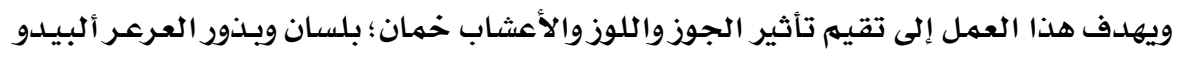

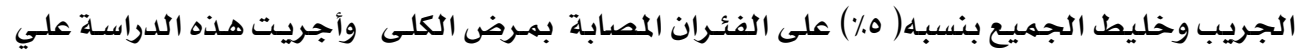

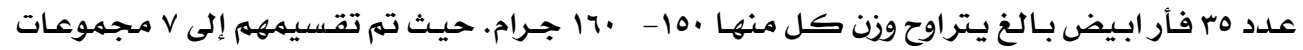

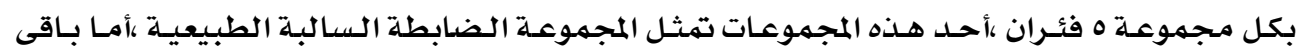

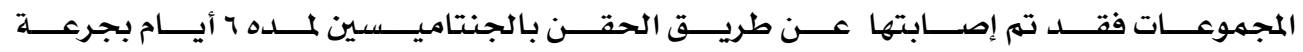

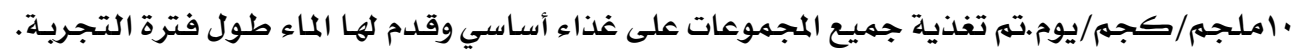

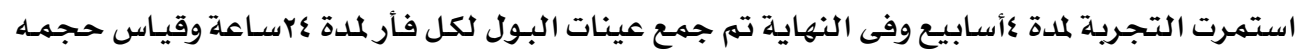

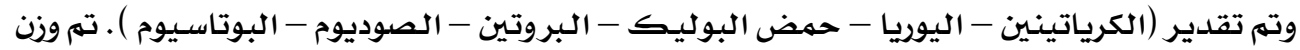

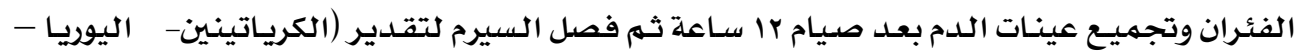

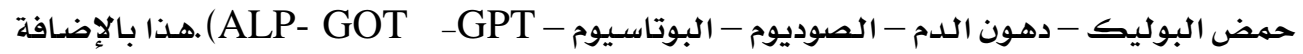

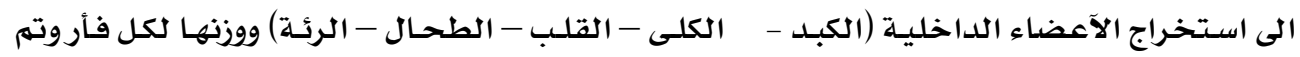

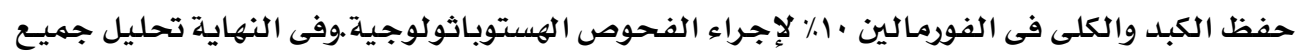

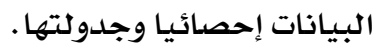

